\title{
Convulsions: Eclampsia or Malaria or Both!
}

\author{
${ }^{1}$ PB Jaju, ${ }^{2}$ Shruti B Bhavi
}

\begin{abstract}
Introduction: Prevalence of malaria in pregnancy is $1.4 \%$. A high index of suspicion is most important in the diagnosis of malaria and should be differentiated from other complications like eclampsia, intrauterine sepsis.
\end{abstract}

Case report: Gravida 3 para 2 living $1\left(\mathrm{G}_{3} \mathrm{P}_{2} \mathrm{~L}_{1}\right)$ with 28 weeks gestation was referred with high blood pressure (BP), severe anemia. She was afebrile, severe pallor + , pedal edema + , pulse rate $(\mathrm{PR})-110 / \mathrm{min}, \mathrm{BP}-140 / 80 \mathrm{~mm} \mathrm{Hg}$, per abdomen (P/A) Ut 28 to 30 weeks size relaxed with fetal heart sounds (FHS) regular. Hemoglobin (HB) $5.3 \mathrm{gm} \%$, platelet count 80,000 . After 3 days, patient threw one convulsion. Low dose $\mathrm{MgSO}_{4}$ regime given. Peripheral smear (PS) for malarial parasite (MP) vivax positive and injection quinine $1200 \mathrm{mg}$ in $10 \%$ dextrose started. She was induced and delivered.

Discussion: In pregnancy malaria is more common, more atypical, more severe and more fatal. Once diagnosed treatment should be started immediately and pregnant woman should be given full doses of antimalarials. Both eclampsia and malaria are to be treated.

Keywords: Cerebral malaria, Convulsions, Plasmodium vivax.

How to cite this article: Jaju PB, Bhavi SB. Convulsions: Eclampsia or Malaria or Both! J South Asian Feder Obst Gynae 2015;7(3):245-246.

Source of support: Nil

Conflict of interest: None

Date of received: 6 August 2015

Date of acceptance: 20 November 2015

Date of publication: December 2015

\section{INTRODUCTION}

Malaria is endemic in India and constitutes a major public health challenge. Prevalence of malaria in pregnancy is $1.4 \%{ }^{1}$ Pregnant women, particularly in 2 nd and 3rd trimesters of pregnancy, are more likely to develop severe malaria then other adults and have a higher malaria related mortality rate ranging from 100 to over 1000/100,000 live births, ${ }^{2}$ cerebral malaria, intrauterine fetal death, preterm labor, pulmonary edema/acute

\footnotetext{
${ }^{1}$ Professor and Head, ${ }^{2}$ Assistant Professor

${ }^{1}$ Department of Obstetrics and Gynecology, Shri BM Patil Medical College, Bijapur, Karnataka, India

${ }^{2}$ Department of Obstetrics and Gynecology, Gadag Institute of Medical Sciences, Gadag, Karnataka, India
}

Corresponding Author: Shruti B Bhavi, Assistant Professor Department of Obstetrics and Gynecology, Gadag Institute of Medical Sciences, Gadag-582101, Karnataka, India, Phone: 9480407256, e-mail: drshrutibhavi@gmail.com respiratory distress syndrome (ARDS), low-birth weight and rarely congenital malaria. In India, Plasmodium falciparum is responsible for the majority of malaria episodes in pre-gnant women (approximately 2/3rd) with remainder due to plasmodium vivax. ${ }^{1}$ Recurrent attacks of $P$. vivax infection during pregnancy can result in significant anemia and low birth weight. ${ }^{3}$

A high index of suspicion is the most important element in the diagnosis of malaria. The risk of death from severe malaria is greatest in first 24 hours. ${ }^{4}$ Malaria is frequently missed or misdiagnosed in pregnancy and needs to be differentiated from complications of pregnancy, e.g. intrauterine sepsis, eclampsia or pyelonephritis, as signs and symptoms may be similar. Cerebral malaria is a diffuse encephalopathy associated with seizures which can occur in up to one third of patients with severe malaria, usually secondary to $P$. falciparum infection. There are very few documented studies on $P$. vivax malaria in pregnancy. It appears to be more common in primigravida, than in multigravidae. However, there are infrequent reports of cerebral malaria associated with $P$. vivax infections.

\section{CASE REPORT}

Mrs XY, aged 27 years, gravida 3 para 2 living $1\left(\mathrm{G}_{3} \mathrm{P}_{2} \mathrm{~L}_{1}\right)$ with 28 weeks period of gestation was referred to BLDE Hospital with a high BP recording and severe anemia. She had $h / o$ loose stools and cough and was on treatment for it. She had no h/o fever, chest pain. No h/o headache, blurring vision, epigastric pain. She had a obstetric history of two previous normal deliveries with one living 4 years old child.

On examination, her weight was $52 \mathrm{~kg}$, she was afebrile, severely anemic, pedal edema+, pulse rate 110/ $\mathrm{min}$, respiratory rate $(\mathrm{RR})-38 \mathrm{cpm}, \mathrm{BP}-140 / 80 \mathrm{~mm} \mathrm{Hg}$, respiratory system (RS) $-\mathrm{B} / \mathrm{L}$ crepts+, cardiovascular system-no abnormality detected (CVS-NAD), P/A-Ut 30 weeks size relaxed with fetal heart sounds $140 / \mathrm{min}$ regular, no organomegaly. Neurological examination revealed normal reflexes.

\section{Lab Investigations revealed}

Hemoglobin $5.3 \mathrm{gm} \%$, total count (TC)-4300 cumm, DC-N 69 L 39, platelet count 80,000. Urine albumin 1+, blood urea $28 \mathrm{mg} / \mathrm{dl}$, serum uric acid $8 \mathrm{mg} \%$, S. creatinine $1 \mathrm{mg} \%$, liver function test (LFT) normal, peripheral smear showing dimorphic anemia. She was suspected of dengue but dengue test was negative. 
In view of anemia, she was transfused with 3 pints of blood followed by IV iron sucrose for 3 doses. After 3 days of admission, patient had c/o nausea and patient threw one episode of convulsion followed by drowsiness. She had fever of $38^{\circ} \mathrm{C}$, pulse rate (PR) $-146 / \mathrm{m}$, $\mathrm{BP}-148 / 80 \mathrm{~mm} \mathrm{Hg}$, glucose random blood sugar (GRBS)-127 mg/dl, low dose $\mathrm{MgSO}_{4}$ regime $4 \mathrm{gm} \mathrm{IV}$ and $3 \mathrm{gm}$ intramuscular (IM) on each buttock given as loading dose and maintenance dose 3 gm IM alternatively 4 th hourly was given and continued for 24 hours. Peripheral smear for malarial parasite (MP) showed $P$. vivax positive and malaria treatment injection quinine $1200 \mathrm{mg}$ in $10 \%$ dextrose $170 \mathrm{ml}$ three times daily (TID) in divided doses over 4 hours was started along with $25 \%$ dextrose $100 \mathrm{ml}$ TID with blood glucose monitoring every 4 to 6 hourly. This treatment was continued for 5 days.

On the same day after 6 hours, patient had a 2nd episode of convulsion along with frothing lasting for 10 seconds. Labor was induced with $25 \mu \mathrm{g}$ misoprostol per vaginally and she delivered a live $1.54 \mathrm{~kg}$ baby with Apgar score 6/10 and 8/10. Postdelivery 1 more pint blood was transfused. Placenta was sent for histopathology report (HPR). It revealed no parasites. Cord blood was checked and was found negative for malaria parasite. After 2 days of delivery, patient was again behaving irrelevant, aggressive and threw a convulsion. Injection diazepam IV given and oral antimalarials tablet quinine $300 \mathrm{mg}$ twice daily (BD) continued for 5 days. Patient recovered uneventfully.

\section{DISCUSSION}

Malaria in pregnancy is a obstetric, social and medical problem requiring multidisciplinary and mutidimensional solution.

Pregnant women constitute the main adult risk group for malaria. Malaria and pregnancy are mutually aggravating conditions. The physiological changes of pregnancy and pathological changes due to malaria have a synergistic effect on the course of each other, thus making the life difficult for the mother, child and treating physician.

Plasmodium falciparum malaria can run a turbulent and dramatic course in pregnant women. The nonimmune primigravida are usually the most affected. In pregnant women the morbidity due to malaria includes anemia, fever, hypoglycemia, cerebral malaria. The problems of newborn include, low-birth weight (LBW), prematurity, intrauterine growth restriction (IUGR) and rarely congenital malaria. In pregnancy, malaria is more common because of immunosuppression; more atypical due to hormonal, immunological and hematological changes; more severe due to high parasitemia and more fatal.

\section{PATHOPHYSIOLOGY}

The pathophysiology of malaria in pregnancy is great due to altered immunity and availability of new organ 'placenta'. A dramatic breakdown of acquired immunity occurs in pregnancy, especially in primigravida.

Plasmodium falciparum has unique ability of cytoadhesion. Chondroitin sulfate A and hyaluronic acid have been identified as the adhesion molecules for parasite attachment to placental cells. The parasites sequester along the surface of placental membrane, trophoblastic villi, fill up the intervillous spaces interfering with oxygen and nutrient transport to the fetus. All the placental tissue exhibit malarial pigments (with or even without parasites).

In areas of high transmission of malaria mortality is less common, asymptomatic and incidental parasitemia are not uncommon and peripheral blood may be negative for malarial parasite whereas in areas of low transmission the risk of malaria infection is greater and can result in maternal death and spontaneous abortions in up to $60 \%$ of cases. Severe malaria occurs when malaria infections are complicated by serious organ failures or abnormalities in the patients blood or metabolism.

If a pregnant woman presents with convulsions, it is necessary to determine whether they are due to malaria or eclampsia. The risk of death from severe malaria is greatest in the first 24 hours. ${ }^{4}$ Once diagnosed treatment should be started immediately and pregnant woman should be given full doses of antimalarials. ${ }^{4}$

In this case, patient presented with fever, high BP, convulsions, urine albumin $1+$, high serum uric acid and smear positive for vivax malaria. She underwent both antimalarial treatment and antieclamptic treatment and pregnancy was terminated.

\section{CONCLUSION}

Eclampsia with cerebral malaria is an acute and dangerous obstetrical disease. Complex therapy with both antimalarial and anticonvulsant treatment should be given to the patients. Termination of pregnancy is the key to effective cure of the disease.

\section{REFERENCES}

1. Brooks MI, Singh N, Hamer DH. Control measures for malaria in pregnancy in India. Ind J Med Res 2008 Sept;128(3):246-253.

2. Detels R, Beaglehole R, Lansang MA, Gulliford M. Chapter 9.15. Malaria. Morrow RH and Moss WJ. Oxford Text book of public health. 5th edition. Oxford Publications 2009;3:1239.

3. Nosten F, McGready R, Simpson JA, Thwai KL, Balkan S, Thein Cho, et al. Effect of plasmodium vivax malaria in pregnancy. The Lancet 1999 Aug;354(9178):546-549.

4. WHO reference manual for healthcare providers. 2nd ed. Prevention and control of malaria in pregnancy; 2008. 